\title{
Invited Commentary: In a Genomic Era, Should We Promote Dopamine Homeostasis to Treat Opiate/ Opioid Abuse, Instead of Blocking Brain Dopamine Function?
}

\author{
Authors: \\ Kenneth Blum*1-8,11,12, Edward J. Modestino, ${ }^{9}$ Roger L. Waite ${ }^{4}$, Eric R. Braverman ${ }^{5}$, \\ Mona $\mathrm{Li}^{5}$, Lyle Fried ${ }^{7}$, Marcelo Febo, ${ }^{1}$ David Baron ${ }^{2}$, Bruce Steinberg ${ }^{9}$, \\ Thomas McLaughlin $^{10}$, B. William Downs ${ }^{11}$, and Rajendra D. Badgaiyan ${ }^{12}$
}

\section{Authors Note:}

${ }^{1}$ Department of Psychiatry \& McKnight Brain Institute, University of Florida College of Medicine, Gainesville, FL, USA; ${ }^{2}$ Department of Psychiatry \& Behavioral Sciences, Keck School of Medicine of USC, Los Angeles, CA, USA; ${ }^{3}$ Division of Applied Clinical Research \& Education, Dominion Diagnostics, LLC, North Kingstown, RI, USA; ${ }^{4}$ Department of Precision Medicine, Sanus Biotech, LLC, Austin, TX, USA; ${ }^{5}$ Division of Clinical Neurology, PATH Foundation NY, New York, NY, USA; ${ }^{6}$ Division of Personalized Medicine, IGENE, LLC, Austin, TX, USA; ${ }^{7}$ Division of Neuroscience Research and Addiction Therapy, Shores Treatment \& Recovery Center, Port Saint Lucie, FL, USA; ${ }^{8}$ Department of Clinical Psychology and Addiction, Eötvös Loránd University, Hungary; ${ }^{9}$ Department of Psychology, Curry College, Milton, MA, USA; ${ }^{10}$ Center for Psychiatric Medicine North Andover, MA, USA; ${ }^{11}$ Department of Nutrigenomics, Victory Nutrition International, LLC, Lederoch, PA, USA; ${ }^{12}$ Department of Psychiatry, Wright State University Boonshoft School of Medicine, Dayton, OH, USA.

Acknowledgements: The Authors appreciate the edits of Margaret A. Madigan and the outstanding support of Mary Hauser of Dominion Diagnostics.

Special Circumstances: Dr. Blum is the owner of US and foreign patents related to KB220Z. US patents include: 6,132,724, 6,955,873, \& EU EP0979092 as well as PCT pending regarding GARS and Kb220.

*Corresponding Author: Kenneth Blum, PhD, Department of Psychiatry \& McKnight Brain Institute, University of Florida College of Medicine, Gainesville, FL, USA; Tel: 352-2944911; Fax: 352-392-9887; Email: drd2gene@gmail.com 


\begin{abstract}
We are currently in a genomics era with important implications for the field of psychiatry. An understanding of DNA, as well as polymorphic changes affecting the brain's reward circuitry, has provided a new way of approaching and thinking about addictive behaviors. Our current goal is to provide a foundation for more accurate genetic diagnoses as well, as the application of dopamine agonist therapy (pro-dopamine regulation), in order to balance dopaminergic activation. Based upon our extensive research, we are proposing a novel approach which challenges the Addiction recovery field to utilize these tools by introducing them into inpatient/outpatient addiction treatment programs The following tools, we hope, will change the recovery landscape: The Genetic Addiction Risk Score (GARS ${ }^{\mathrm{TM}}$ ) for Reward Deficiency Syndrome (RDS) diagnoses; the Comprehensive Analysis Of Reported Drugs (CARD ${ }^{\mathrm{TM}}$ ) to establish compliance of prescribed medications and abstinence during treatment; natural Dopamine agonistic therapy $\left(\mathrm{KB} 220^{\mathrm{TM}}\right)$; mRNA (patent pending) to resolve pre-and postcandidate gene expressions in Reward Deficiency Syndrome (RDS). As a result, we have dubbed this paradigm shift as: "The Reward Deficiency Solutions System (RDSSTM)."
\end{abstract}

Keywords: Genome, Genetic Addiction Risk Score (GARS), KB220 variants, Pro-Dopamine Regulation, Pain, Opiate/opioid epidemic 


\section{Introduction: Opiate/Opioid Epidemic in America}

The abuse of and addiction to opioids, such as heroin, morphine, and prescription pain relievers is a global problem affecting the health, social, and economic welfare of all societies. It is estimated that between 26.4 million and 36 million people abuse opioids worldwide (UNODC, 2012) with an estimated 2.1 million people in the United States suffering from substance use disorders (SUDs) related to opioid pain relievers in 2012 and an estimated 467,000 addicted to heroin (SAMHSA, 2013). The consequences of this abuse have been devastating and are rising. For example, the number of unintentional, overdose deaths from prescription pain relievers has soared in the United States, more than quadrupling since 1999. There is also growing evidence suggesting a relationship between increased, non-medical use of opioid analgesics and heroin abuse (Muhuri, Gfroerer, \& Davies, 2013).

Deaths related to prescription opioids began rising in the early $21^{\text {st }}$ century. By 2002, death certificates listed opioid analgesic poisoning as a more common cause of death than heroin or cocaine (Paulozzi, Budnitz, \& Xi, 2006): In 2012, over five percent of the U.S. population aged 12 years or older used opioid pain relievers non-medically (CBHSQ, 2013). The public health consequences of opioid pain reliever abuse are disturbingly widespread. For example, abuse of prescription pain relievers by pregnant women can result in a number of problems in newborns, referred to as neonatal abstinence syndrome (NAS). This diagnosis has increased by almost 300 percent in the United States between 2000 and 2009 (CDC, 2013), largely driven by the high rate of opioid prescriptions provided to pregnant women. In the United States, an estimated 14.4 percent of pregnant women are prescribed an opioid during their pregnancy (Bateman et al., 2014).

While NIDA supports Medication Assisted Treatment (MATs), especially Methadone and Buprenorphine as important treatment options for opiate/opioiddependent patients, that institution also suggests that their use is underutilized. It is our contention, that, while these drugs are useful in the short term, they lock people into addiction. As a result, we are proposing that MATs eventually be replaced with other more useful options.

Drugs of abuse/addiction act by attaching to specific proteins, called opioid receptors, which are found on nerve cells in the brain, spinal cord, gastrointestinal tract, and other bodily organs. When these substances attach to their receptors, they reduce the perception of pain and can also produce a sense of well-being. On the other hand, they can also produce drowsiness, mental confusion, nausea, and constipation (Mattoo, 2009). The effects of opioids are typically mediated by specific subtypes of opioid receptors (i.e., mu, delta, and kappa), which are activated by the body's own (endogenous) opioid chemicals (i.e., endorphins, enkephalins). With repeated administration of opioid drugs (prescription or heroin), the production of endogenous opioids is inhibited, accounting for, in part, the discomfort that ensues, when the drugs are discontinued (i.e., withdrawal). Adaptations of these opioid receptors' signaling mechanism have also been shown to contribute to withdrawal symptoms. 


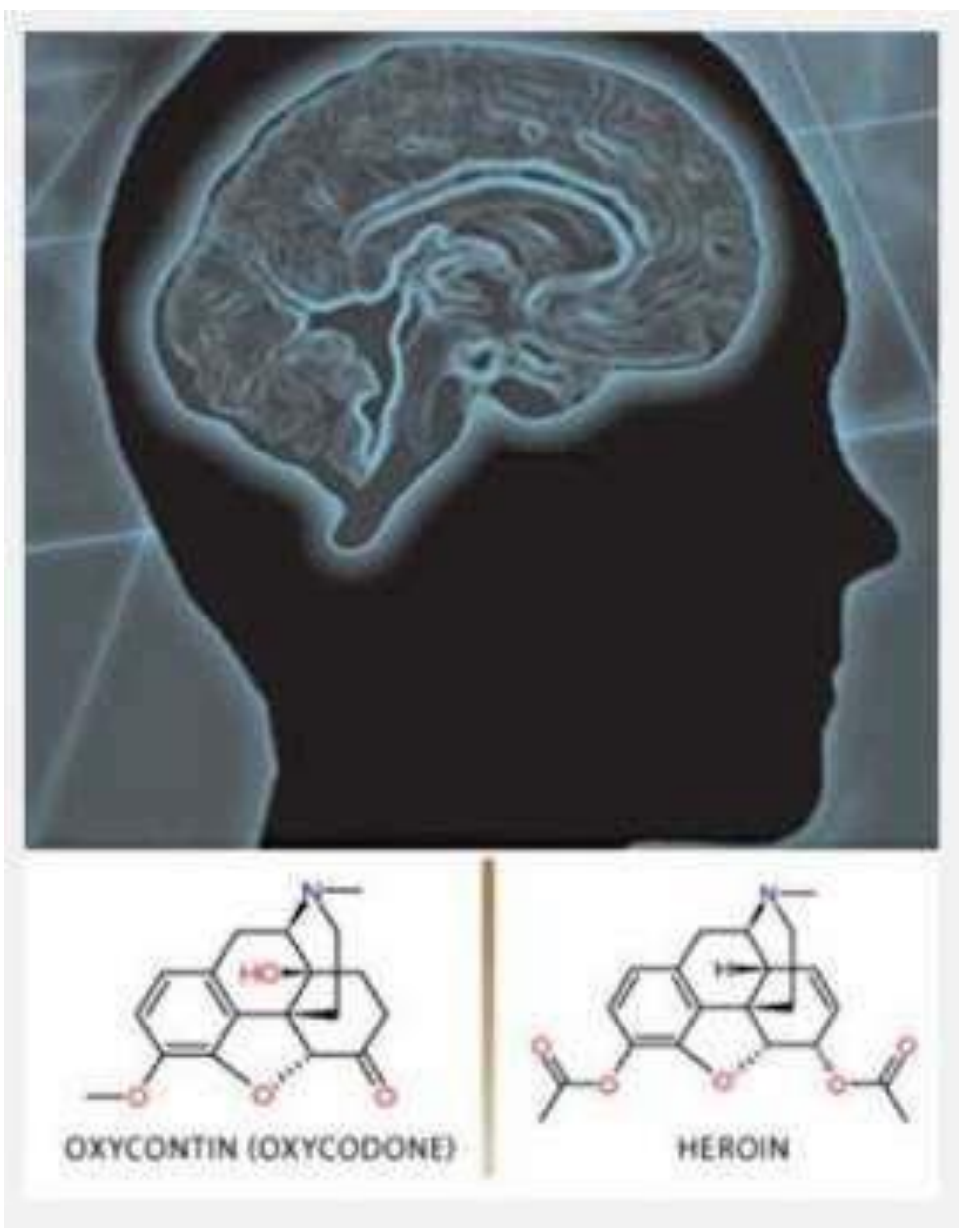

Figure 1. Structural Formulae of opioids (taken from online)

\section{Genesis of Behavior and Appropriate Diagnosis}

The genesis of all behaviors, be they "normal" (acceptable) or "abnormal" (socially unacceptable), derives from an individual's genetic makeup at birth. This genetic predisposition, derived from multiple gene combinations and polymorphisms, is expressed differently based on numerous environmental factors, including family, friends, educational and socioeconomic status, environmental contaminant exposure, and the availability of psychoactive drugs, including food. The main predisposition for these behaviors derives from a set of genes, which promote a feeling of well-being via neurotransmitter interaction at the "Reward Center" of the brain. This site is located in the meso-limbic system and its stimulation leads to normal dopamine release.

Subjects afflicted with RDS carry polymorphic genes affecting their dopaminergic pathways which result in hypo-dopaminergic function. The latter is caused by a reduced number of dopamine D2 receptors, reduced synthesis of dopamine (by interference with the enzyme, dopamine beta-hydroxylase), reduced net release of pre-synaptic dopamine (from, e.g., the dopamine D1 receptor), increased synaptic 
clearance due to a high number of "dopamine transporter" sites and low dopamine (D2) receptor densities rendering such people more vulnerable to addictive behaviors (Blum et al., 2000). The RDS rubric adumbrates shared genes and behavioral tendencies, including, dependence on: alcohol, psycho-stimulants, marijuana, nicotine (smoking), and opiates (altered opioid receptor function), carbohydrate issues (e.g., sugar-binging), obesity, pathological gambling, sex addiction, premeditated aggression, stress, pathological aggression, certain personality disorders, including novelty- seeking and sex addiction, and even internet addiction. The common factor across all of these substances and behaviors is that they induce presynaptic dopamine release and, thus, ameliorate RDS cravings (Blum \& Gold, 2011).

Spectrum disorders, such as ADHD, Tourette's syndrome, and Autism are also thought to be related to dopamine dysregulation. Very few behaviors, including anhedonia however, depend upon the effects of a single gene. Complexes of genes (polygenic) drive most hereditary human actions, and RDS is no exception.

For example, DRD2 and DAT1 gene polymorphisms are reliably associated with reward-dependent traits, such as cocaine dependence. As a polygenic disorder, involving multiple genes and polymorphisms, RDS likely requires a threshold number of RDS-associated polymorphisms in order to become manifest in a particular subject. Unaffected individuals in the population also carry some of these alleles, with, for example the dopamine D2 receptor gene A1 allele being present in about one-third of unscreened Americans (Noble, 2003).

The preceding findings have implications for both the diagnosis and targeted treatment of RDS behaviors, after analyzing the presence of dopaminergic genetic polymorphisms. Without being bound to a particular theory, but in keeping with the concept of common, neuro-genetic mechanisms, we believe that RDS is a basic phenotype, covering many reward-related behaviors and psychiatric disorders, including the so-called spectrum disorders (Blum, Thanos et al., 2015).

We are proposing that a polygenic genetic Addiction Risk Score "GARS" test can be used, to determine stratified, genetic risk of patients with addictive behaviors, upon their entry into a treatment facility, by providing information about an individual's genetic predisposition to RDS, based on an analysis of a number of RDS-associated alleles they carry (Blum, Badgaiyan, et al., 2015).

Approximately $0.1 \%$ (1 in 1,000) nucleotides differ between any two copies of the human genome. Some of these genetic variations, referred to as "single nucleotide polymorphisms" or "SNPs", lead to differences in the proteins encoded by genes. Others are "silent", residing in non-protein coding regions of the genome. SNPs are used, for example, to diagnose genetic disorders (e.g., Huntington's disease, Alzheimer's disease, etc.), to determine predisposition to disease (e.g., various forms of cancer), identify or determine the ancestry of a genetic sample or correlate genetic sequences with phenotypic conditions, such as drug response and toxicity (Galvan, André, Wang, Cepeda, \& Levine, 2012). 
Single nucleotide polymorphisms can be identified in nucleic acid samples, by any suitable method, such as DNA sequencing, restriction enzyme analysis or site-specific hybridization. Flow-throughput genomewide screening for SNPs requires the ability to simultaneously analyze multiple loci with high accuracy and sensitivity. The current "state of art" utilizes multiplex assays to detect one or many RDS-associated SNPs. These RDS-associated SNPs can be identified by any suitable method, including DNA sequencing of patients, diagnosed with one or more RDS behaviors. Once identified and validated, the presence, if any, of one or more RDS-associated SNPs in the nucleic acids derived from a biological sample taken from a patient can be determined via any current suitable or later to-be-developedassay, including ones that rely on sitespecific hybridization, restriction enzyme analysis or DNA sequencing.

We are currently living in a genomics era that should promote exciting medical advancements, particularly in the field of psychiatry. An understanding of DNA and polymorphic associations and their relationship to the brain's reward circuitry has stimulated a new way of approaching and thinking about addictive behaviors. Our goal is to provide the foundations for more accurate, genetic diagnoses as well as the use of dopamine agonist therapy (pro-dopamine regulation) to balance dopaminergic activation.

As a result of our work on the nature of addiction, we are humbly proposing a novel approach and challenging the recovery field to introduce these tools into inpatient/outpatient addiction treatment programs. The following constitute tools that, we hope, will change the Recovery
Landscape: The Genetic Addiction Risk Score (GARS ${ }^{\text {TM}}$ ) and the Blum-Demotrovics RDS Questionnaire for Reward Deficiency Syndrome (RDS) diagnoses; the Comprehensive Analysis Of Reported Drugs (CARD $^{\mathrm{TM}}$ ) to establish compliance of prescribed medications and abstinence during treatment; natural Dopamine agonistic therapy (KB220 $\left.{ }^{\mathrm{TM}}\right)$; and, finally, mRNA (patent pending) to resolve pre- and post-candidate gene expressions in RDS. As a result, we name this paradigm shift: "Reward Deficiency Solutions System (RDSS ${ }^{\mathrm{TM}}$ )" (Blum, Febo et al., 2014).

3. Why are so many Americans and individuals across the globe falling victim to the chain of addictive behaviors and becoming part of one of the worst epidemics in the history of the world?

The answer to this question is, in part, both genetic and environmental (epigenetic). For example, recently, researchers have found that epigenetic effects on the chromatin structure of our DNA are a legacy passing on from generation to generation. Scientists such as Stephen Hawkins suggest that humans are made up of self-assembled molecules, generated over 14 billion years ago. Perhaps, even more interesting is the fact that as members of Homo sapiens, individuals differ from each other in their DNA by only $0.5 \%$ (Gokcumen et al., 2013). Recent evidence shows that each human has an average of 60 new mutations, compared to his/her parents. Even more remarkable is the fact that the human brain contains billions of neurons, working in concert, to provide us the gift of "well-being," free of mental disease and stress. The number of neurons in the brain varies dramatically from species to species. One estimate estimates 
that the human brain has about 85 billion neurons and, approximately, 85 trillion synapses. To support this system, $20 \%$ of the body's energy is consumed in order to keep the brain working normally.

The differences between individual humans are 4.25 billion neurons and 4.25 trillion synapses that make us unique. This difference affects the 7.4 billion humans roaming the earth, as we work and live together trying to achieve some degree of productivity and happiness. This is responsible for cultural diversity, whereby both genetic make-up and epigenetics shape our behavioral phenotype. However, as the world turns, $21^{\text {st }}$ century humans are faced daily with acts of terrorism and horrific diseases that arise because many of these genetic and epigenetic differences lead to fatalities, not just from cancer, but from mental impairments influencing billions of neurons and trillions of synapses. The particular molecular rearrangement of our genome makes each of us unique. In addition, the way dopamine functions in our Reward System may also be unique. One example, among other gene variations involved in brain reward, is the finding that genetic differences account for the presence of Attention Deficit/Hyperactivity Disorder (ADHD), a subtype of RDS, in approximately $8-12 \%$ of children in the United States and $4 \%$ of adults worldwide (Gold, Blum, Oscar-Berman, \& Braverman, 2014).

In addition, at birth, an estimated 110,000 million Americans carry a gene form (allele) of a single genetic variation involving the brain's dopamine D2 receptors. It has been established, for example, that carriers of the allele DRD2 Taq A1 have 30-40\% less D2 receptors in their brains (Noble, Blum, Ritchie, Montgomery, \& Sheridan, 1991). What are the implications of this finding with respect to our romance with getting high - "turning on" and "turning off" via the use of potent psychoactive drugs (e.g., alcohol, cocaine, and opiates) and the resultant addiction and fatalities seen in children?

To help us understand the complex interaction of genetic polymorphisms and resultant predisposition for all addictions, we must discuss the confirmed psychiatric genetic experiment performed in 1990. Specifically, the first association of an allelic variant (A1) of the dopamine D2 receptor gene (DRD2) and severe alcoholism was discovered by Blum et al. (1990). Later, confirmatory experiments showed that individuals, who carry this variant, have 30$40 \%$ lower dopamine receptors than DRD2 A2 carriers (Noble, Blum, Ritchie, Montgomery, \& Sheridan, 1991; Pohjalainen et al., 1998). Simply put, being born with this single gene variation (DRD2 A1 form) known to cause low dopamine D2 receptors, renders an individual with a high addiction risk (vulnerability) to any substance or behavior that stimulates the neuronal release of dopamine. In fact, in 1996, Blum's laboratory used a mathematical model (Bayesian Theorem) to predict that an individual born with the A1 allele (variant) has a $74.4 \%$ risk of developing a RDS behavior, viz., addiction (Blum, Wood, Braverman, Chen, \& Sheridan, 1995). Individuals with this allele typically have an initial acute response to the use of a psychoactive drug, engaging in pathological gambling, or whatever behavior stimulates enough neuronal dopamine in order for them to feel normal, possibly, for the first time. Unfortunately, chronic consumption/ 
experiences leads to epigenetic changes that further reduce dopamine receptor numbers, resulting in a stronger need to abuse, followed by additional, unwanted and uncontrollable behaviors and, even, fatal narcotic overdose.

\section{How were the genes involved in reward found?}

Chemical messengers (neurotransmitters) in the brain are like keys that turn on various functions of genes. The neurotransmitters that participate in evoking pleasurable feelings in the reward circuitry work in a cascading fashion throughout the brain. These interactions ("Brain Reward Cascade") may be viewed as activities of subsystems within a greater system, occurring simultaneously or in sequence and merging in cascade-like fashion toward a particular effect (Blum, Febo, Badgaiyan, et al., 2016). The biological goal is the generation of feelings of well-being via the eventual release of just the right amount of dopamine at the reward site. In this scenario, there are at least seven major neurotransmitters classes that are involved: serotonin, cannabinoids, endorphins (enkephalins), GABA, glutamate, acetylcholine and dopamine. Thousands of published studies involve these reward genes and pathways, influencing the function of these neurotransmitters. This research has involved the identification of gene (DNA) variations or alleles that individuals are born with as well as epigenetic (environmental RNA) changes that may alter the healthy, intended function of DNA (Damez-Werno et al., 2016). In simpler terms, Nestler's group showed that if histone arginine methylation is blocked on the dopamine D2 receptor gene following cocaine-induced epigenetics, the resultant dopamine signaling at the reward site [Nucleus Accumbens (Nac)] functions as a homeostatic brake to restrain cocaine action. This blocked methylation will lead to enhanced mRNA expression of the $\mathrm{D} 2$ receptor. This finding could provide novel candidates for the development of treatments for cocaine addiction and supports the concept of "Pro-Dopamine Regulation" as a therapeutic modality.

Dysfunctional DNA is due to, what is referred to as, single nucleotide polymorphisms, frequently, also known as SNPS. SNPS are the most frequent kind of genetic variation among individuals. Each SNP constitutes a variance in a single DNA building block or nucleotide. For example, a SNP may substitute the nucleotide cytosine I with the nucleotide thymine (T) in a specific strand of DNA. SNPs typically occur throughout one's DNA. SNPs occur once every 300 nucleotides on average, which translates to approximately 10 million SNPs in the entire human genome. Usually, these variations can be located in the DNA between genes. They can behave as biological markers, assisting scientists to find genes linked to disease. When SNPs occur within a gene or near a gene (in a regulatory region) they alter the gene's function. If these SNPS are found in the Brain Reward Cascade's [BRC] set of genes (CDC, 2013), the neurotransmission will be dysfunctional resulting in a loss of dopamine regulation or balance (homeostasis). Too little dopamine will, at birth, predispose individuals to "want" or "like" psychoactive drugs or even behaviors like hyper-sexuality and gambling. Compromised DNA with risk variations (alleles) can predispose them to become victims to chain of addictive behaviors (Blum, Downs et al., 2016). 
Following 25 years of worldwide research, a panel of ten Reward Gene Risk Variants and as well as 11 polymorphisms, called the Genetic Addiction Risk Score (GARS), has been developed. In unpublished work, when GARS was compared to Addiction Severity Index (ASI) used in many clinical settings, it was found to reliably predict the severity of both alcohol and drug dependency (Blum, Smolen et al., 2014). In support of the notion of early testing for addiction vulnerability and other RDS subtypes seems parsimonious and may be important even in potential soldiers entering military battle to identify those with a high risk for PTSD. The GARS may also be very informative in identifying subsets of the population such as ultra-high buprenorphine metabolizers in AfricanAmericans yielding pertinent information linked to resource allocation (Home 1 or Home 2).

Parents caught up in today's horrific demographic of 127 people, both young and old, dying from opiate/opioid overdose every day in America, need help. Families in the past would have never imagined that their loved ones would die or be in real danger from opiate addiction. Author Bill Moyers published an article in Parade Magazine in the 90's, wherein he reported as he traveled around the United States, that too many children were diagnosed with ADHD and that many of those children later developed addictions, such as substance dependence. He called for better ways to identify these children and treat them with approaches other than potentially addictive pharmaceuticals.

When the GARS test becomes available and is approved by the FDA, especially, for parents to test their children (and without such approval for clinicians to test their patients), the field will be able to assess the patient's vulnerability for chemical dependency and, more importantly, their children's risk of RDS behaviors like addiction, ADHD, and autism spectrum disorders. The common factor produced by these high-risk gene variants is that they lead to a low dopamine (hypo-dopaminergic) function or deficit.

Arguments against testing, such as the fear of labeling and knowing the risk, are also considerations. The real issue or challenge, however, is what can be done if risk alleles are found? It is understandable that, when there is one gene-one disease (OGOD) involved, such as in Huntington's disease, when treatment is unavailable and prevention is problematic, why know the risk? (Plomin, Owen, \& McGuffin, 1994).

The question is: Have we found a safe, non-addictive/divertible solution that will provide the brain with a means of balancing the neurotransmitters involved in the BRC, culminating in true dopamine homeostasis? In spite of variant genes and epigenetic, environmental insults, holistic approaches like mindfulness, exercise, brain spotting, spirituality and, particularly, amino acid therapy (KB220 formulations), have been shown to reduce relapse and increase brain dopamine homeostasis (Blum, Febo, \& Badgaiyan, 2016).

The authors are suggesting that not only should clinicians in the near future be able to genetically test children for unwanted reward gene risk variants, which predispose them to dopamine deficiency and the lack of reward, putting them at risk for drug and non-drug addictions, but these same clinicians might also be in a position to 
prevent other RDS behaviors noted above. It seems possible that genetic risk for substance abuse and other RDS behaviors may be identifiable by the GARS test and offer an explanation why some individuals are more vulnerable than others

With continued research, genetic and epigenetic dopamine deficiencies can be treated, relapse reduced, and addicts freed from the clutches of powerful addictive behaviors, with a resultant balance and happiness in their lives. This laudable goal requires intensive research.

\section{The Medical Implications of RDS Behaviors}

There are hundreds of mutations and several thousand permutations of these mutations, which can result in, for example, diabetes. This disease was thought of involving an "elevated rise in blood glucose level," beyond certain arbitrary values accepted by certain professional bodies.

An elevation in the blood sugar level is, in fact, only one of the several 'signs' of DMT2 and its correction has been regarded as solely involving reducing this level to an acceptable normal range. We similarly manage most diseases of "modern medicine" by, for example, using antibiotics against certain infections. An additional treatment example from this decade involves the use of "Imatinib" $~($, which is "a designer drug" used in the treatment of multiple cancers, most notably Philadelphia chromosomepositive $(\mathrm{Ph}+)$ chronic myelogenous leukemia. This drug has been designed, based upon insight into the disease's molecular pathology, with a certain degree of precision (Yamaguchi et al., 2016).
On the other hand, there is strong evidence that alcohol causes cancer in at least seven sites in the body. With current estimates suggesting that alcohol-attributable cancers constitute $5.8 \%$ of all cancer deaths world-wide, the question arises as how to best to diagnose and treat not only alcoholism, but all RDS addictions and behaviors.

On the basis of neurochemical and genetic evidence, we suggest that both prevention and treatment of multiple addictions, such as dependence on alcohol, nicotine and glucose, should involve a biphasic approach. Acute treatment should consist of preferential blocking of postsynaptic Nucleus Accumbens [NAc] dopamine receptors (D1-D5), whereas longterm activation of the meso-limbic dopaminergic system should involve activation and/or release of dopamine (DA) at the NAc site.

Failure to follow this model will result in abnormal mood, behavior and potential suicidal ideation. Individuals possessing a paucity of serotonergic and/or dopaminergic receptors as well as an increased rate of synaptic DA catabolism due to high catabolic genotype of the COMT gene are predisposed to self-medicating any substance or behavior that will activate DA release. Such substances and behaviors include alcohol, opiates, psycho-stimulants, nicotine, gambling, sex, and, even, excessive internetgaming including "transfer phenomena".

Acute use of these substances and/or stimulatory behaviors induce a feeling of well-being. However, sustained abuse leads to a toxic "pseudo-feeling of well-being", resulting in tolerance, disease, discomfort or anhedonia (Eisenstein et al., 2016). Thus, a 
reduced number of DA receptors, due to the presence of the DRD2 A1 allelic genotype results in excessive craving; whereas a normal or sufficient amount of DA receptors results in low craving.

To prevent substance abuse, one goal would be to induce a proliferation of DA D2 receptors, in genetically prone individuals. While in vivo experiments using a typical D2 receptor agonist such as bromocriptine induce down regulation, experiments in vitro have shown that constant stimulation of the DA receptor system via a known D2 agonist results in significant proliferation of D2 receptors, in spite of genetic antecedents. In essence, D2 receptor stimulation signals negative feedback mechanisms in the mesolimbic system to induce mRNA expression causing proliferation of $\mathrm{D} 2$ receptors.

\section{Have we hatched DNA- customized nutrition?}

Considering these hypothesisgenerating studies, as well as the paucity of research, we designed a study to assess the practice of DNA customization via the use of a nutritional solution for both wellness and weight management. Blum's group reviewed several studies [30-32], in which their laboratory genotyped 1,058 subjects and administered to these subjects a prodopamine regulator, KB220Z [formerly LG9939, Recomposize, Genotrim] (which is a complex Neuroadaptagen nutraceutical containing-d-l-phenylalanine, chromium, 1tyrosine as well as other select amino-acids and adaptogens) based on polymorphic outcomes. The resulting, customized formulae targeted a minimum of 175 Single Nucleotide Polymorphisms (SNPs), across 16 genes important to the BRC and, which were, most importantly, involved in "dopamine homeostasis."

In a small subset, t-tests comparing various parameters before and after 80 days on the nutraceutical were carried out. The meaningful clinical results are the following: weight loss ( $p<0.008$ ); decrease in sugar craving ( $\mathrm{p}<0.008)$; appetite suppression ( $\mathrm{p}$ $<0.004)$; decrease in snacking ( $\mathrm{p}<0.005)$; decrease in late night binging $(\mathrm{p}<0.007)$; increased awareness of over-eating ( $\mathrm{p}<$ 0.02); improved energy ( $\mathrm{p}<0.004)$; improvement of sleep quality $(\mathrm{p}<0.02)$; and increase in happy emotions $(\mathrm{p}<0.02)$.

Polymorphic correlates were also acquired for several genes (PPAR gamma 2, MTHFR, 5-HT2a, and DRD2 genes) with positive clinical factors tested in this study. Notably, of all the results and gene polymorphisms tested, only the DRD2 gene polymorphism (A1 allele) had a significant Pearson correlation with treatment days $(\mathrm{r}=$ $0.42, p=0.045$ ), so that there was a modest relationship between the occurrence of the A1 allele and the length of treatment days This 2-fold increase is a significant genotype for treatment compliance (Blum, Chen et al., 2006, 2007, 2008). [A correlation coefficient of .42 indicates that the square of .42 or about only $16 \%$ of the variance of one factor is accounted for by the other factor; the statistically significance level only means that the likelihood of a chance finding was less that 1 out of $20(\mathrm{p}<.05)$.] Additionally, Blum's team assessed the effect of the polymorphisms of these five candidate genes as critical targets for the design of a DNA customized nutraceutical, $\mathrm{KB} 220 Z$, to combat obesity. Body recomposition served as the dependent variable as measured by Body Mass Index (BMI) (Blum, Chen et al., 2008). A total of 
21 individuals were evaluated in a this preliminary, investigational study of KB220Z.

The investigation used the outcomes of buccal swab genotyping for each subject. An individualized, customized nutraceutical formula was use based upon measured gene polymorphisms of five gene candidates. At the beginning of the investigation and every two weeks thereafter, each subject completed an adapted Blum-Downs OPAQuE Scale ${ }^{\mathrm{TM}}$ (Overweight Patient Assessment Questionnaire). The alleles involved were: the DRD2; MTHFR C 677T; 5HT2a 1438G/A; PPAR- $\gamma$ Prol2Ala; and Leptin Ob1875 < 208bp. Pre- and post-hoc analyses indicated a difference between the initial BMI and the BMI means, following an average of 41 days (28-70 d) of KB220Z ingestion by the 21 subjects. The pre-BMI mean value was 31.2 (weight/Ht2), compared to the post-BMI mean of 30.4 (weight/Ht2) $\quad(\mathrm{p}<0.034 \quad$ (one-tailed). Comparably, the mean pre-test weight in pounds (lb) was 183.52, compared to the mean post-test weight of $179 \mathrm{lb}$, ( $\mathrm{p}<.047$ ). The results also indicated trends in the decrease of late-night snacking, carbohydrate craving, stress, and waist circumference. Furthermore, within the 41day time frame, these authors observed a trend in weight loss, with $71.4 \%$ of the subjects having lost weight. In particular, 15 of 21 subjects lost weight, with a $\mathrm{z}$ score of $2.4(\mathrm{p}<0.02)$. In this group, 53\% lost an average of over $2.5 \%$ of their initial weight. Further findings, which point to the need for additional research, using a Path Analysis (non-customized KB220Z), also indicated significant connections with anti-obesity behaviors. In a one-year cross-sectional, open trial study of 24 unscreened subjects, using an oral $\mathrm{KB} 220 \mathrm{Z}$ variant, the following beneficial outcomes occurred: decreased stress; sleep improvement; increased energy; universal well-being; decreased cravings (sugar/carbohydrates); enhancement in mental focus/memory; enhancement in blood sugar levels; decreased food intake; decreased waist circumference; weight loss; decreased blood pressure; enhanced exercise performance; decreased drug-seeking; decreased hyperactivity; and decreased cholesterol levels (Blum, Chen et al., 2006).

Although opioid medications effectively treat acute pain and help relieve chronic pain for some patients (Moore, Derry, Eccleston, \& Kalso, 2013), their addiction risk presents a dilemma for healthcare providers seeking to relieve suffering, while preventing drug abuse and addiction. Little is currently known about the risk for addiction among patients treated for chronic pain or about how basic pain mechanisms interact with prescription opioids to influence addiction potential.

To better understand this question, NIDA has launched a research initiative on "Prescription Opioid Use and Abuse in the Treatment of Pain." This initiative program encourages a multi-disciplinary approach using both human and animal studies to examine factors (including pain itself) that predispose to or protect against opioid abuse and addiction. Funded grants cover clinical neurobiology, genetics, molecular biology, prevention, treatment, and services research. This information should help develop screening and diagnostic tools that physicians can use to assess the potential for prescription drug abuse in their patients. Because opioid medications are prescribed for all ages and populations, NIDA is also encouraging research that 
assesses the effects of prescription opioid abuse by pregnant women, children, and adolescents as well as how such abuse in these vulnerable populations might increase the lifetime risk of substance abuse and addiction (Skolnick \& Volkow, 2016). The recently passed "Obama Cure Act" has earmarked one-billion over a two year period to combat the American Opioid epidemic. While the buprenorphine/naloxone model is considered the "Gold Standard" of care, its long-term benefits must be questioned because this treatment option locks patients into addiction.

\section{Can Pro-Dopamine Regulation Assist in Pain Control?}

Another important initiative pertains to the development of new approaches to treat pain. This approach includes research to identify new pain relievers with reduced abuse, tolerance, and dependence risk as well as to devise alternative delivery systems and formulations for existing drugs that minimize diversion and abuse (e.g., by preventing tampering and/or releasing the drug over a longer period of time) and reduce the risk of overdose deaths.

New compounds are being developed that exhibit novel properties as a result of their combined activity on two different opioid receptors (i.e., mu and delta). Preclinical studies show that these compounds can induce strong analgesia but fail to produce tolerance or dependence. Researchers are also closer to developing a new generation of non-opioidbased medications for severe pain that would circumvent the brain reward pathways and, thereby greatly reduce abuse potential. This work includes compounds that work through a type of cannabinoid receptor, found primarily in the peripheral nervous system. NIDA is also exploring the use of non-medication strategies for managing pain. An example is the use of "neurofeedback," a novel modality of the general biofeedback approach, in which patients learn to regulate specific regions in their brains by getting feedback from realtime fMRI brain images. This technique has shown promising results in altering the perception of pain in healthy adults as well as chronic pain patients and could evolve into a powerful psychotherapeutic intervention capable of rescuing the circuits and behaviors impaired by addiction (Borsook et al. 2016).

Along the lines of alternative approaches, such as utilizing the GARS test to diagnose individuals with high or moderate risk for addictive behaviors from strong opioids like Fentanyl and others, restrictions on opioid prescriptions should be implemented and alternatives should become a standard. We are also proposing that the KB220z variant be considered as adjunctive therapy to help raise dopamine tone and reduce pain (Badgaiyan, Sinha, Sajjad, \& Wack, 2015).

\section{Conclusion}

The authors propose that D2 receptor stimulation can be accomplished via the use of KB220 variant, a natural but therapeutic nutraceutical formulation that potentially induces DA release, causing an induction of D2-directed mRNA and, thus, proliferation of D2 receptors in the human. This proliferation of D2 receptors, in turn, should induce an attenuation of craving behavior. In fact, as mentioned earlier, this model has 
been demonstrated to induce a DNAdirected, compensatory over-expression (a form of gene therapy) of the DRD2 receptors, resulting in a reduction in both alcohol-craving behavior in alcoholpreferring rodents and in cocaine selfadministration (Thanos, Michaelides, Umegaki, \& Volkow, 2008; Thanos et al., 2005).

Utilizing natural dopaminergic repletion therapy to promote long term dopaminergic activation will ultimately lead to a common, safe and effective modality to treat RDS behaviors, including SUDs, Attention Deficit Hyperactivity Disorder (ADHD), possibly hypersomnias including narcolepsy (Modestino et al., 2015), obesity and other reward-deficient, aberrant behaviors. This prediction is supported by the more comprehensive understanding of the role of dopamine in the NAc as a "wanting" messenger in the meso-limbic DA system (Blum, Gardner, Oscar-Berman, Gold, 2012). Independent of the future best treatment options including Buprenorphine/ naloxone among others, it is imperative to incorporate genetic testing, both pharmacogenetic (P450system) and pharmacogenomics (GARS), in all patients attending treatment programs.

\section{Author Contributions}

$\mathrm{KB}$ wrote the original manuscript with editorial assistance from EJM, RLW, ERB, ML, LF, MF, DB, BS, TM, BWD, and RDB. EJM formatted the manuscript.

\section{Funding:}

Rajendra D. Badgaiyan is supported by the National Institutes of Health grants 1R01NS073884 and 1R21MH073624; Marcelo Febo is the recipient of R01DA019946 and R21 DA038009. 


\section{References}

Badgaiyan, R.D., Sinha S., Sajjad, M., \& Wack, D.S. (2015). Attenuated tonic and enhanced phasic release of dopamine in Attention Deficit Hyperactivity Disorder. PLoS One, 10(9): e0137326. doi: 10.1371/ journal.pone. 0137326 .

Bateman, B.T., Hernandez-Diaz, S., Rathmell, J.P., Seeger, J.D., Doherty, M., Fischer, M.A., \& Huybrechts, K.F. (2014). Patterns of Opioid Utilization in Pregnancy in a Large Cohort of commercial insurance beneficiaries in the United States. Anesthesiology, 120(5):1216-24. doi: 10.1097/ALN.0000000000000172

Blum, K., Badgaiyan, R.D., Agan, G., Fratantonio, J., Simpatico, T., Febo, M., Haberstick, B.C., Smolen, A., \& Gold, M.S. (2015). Molecular genetic testing in Reward Deficiency Syndrome (RDS): Facts and fiction. J. Reward Defic Syndr., 1(1):65-68.

Blum, K., Braverman, E.R., Holder, J.M., Lubar, J.F., Monastra, V.J., Miller, D., Lubar, J.O., Chen, T.J., \& Comings, D.E. (2000). Reward deficiency syndrome: a biogenetic model for the diagnosis and treatment of impulsive, addictive, and compulsive behaviors. Journal of Psychoactive Drugs, 32 Suppl:i-iv, 1112.

Blum, K., Chen, T.J., Meshkin, B., Downs, B.W., Gordon, C.A., Blum, S., Mengucci, J.F., Braverman, E.R., Arcuri, V., Varshavskiy, M., Deutsch, R., \& Martinez-Pons, M. (2006). Reward deficiency syndrome in obesity: a preliminary cross- sectional trial with a Genotrim variant. Adv Ther., 23(6):1040-51.

Blum, K., Chen, T..J., Meshkin, B., Downs, B.W., Gordon, C.A., Blum, S., Mangucci, J.F., Braverman, E.R., Arcuri, V., Deutsch, R., \& Pons, M.M. (2007) Genotrim, a DNAcustomized nutrigenomic product, targets genetic factors of obesity: hypothesizing a dopamine-glucose correlation demonstrating reward deficiency syndrome (RDS). Med Hypotheses, 68(4):844-52.

Blum, K, Chen, A.L., Chen, T.J., Rhoades, P., Prihoda, T.J., Downs, B.W., Waite, R.L., Williams, L., Braverman, E.R., Braverman, D., Arcuri, V., Kerner, M., Blum, S.H., \& Palomo, T. (2008). LG839: antiobesity effects and polymorphic gene correlates of reward deficiency syndrome. Adv Ther., 25(9):894-913. doi: 10.1007/s12325-008-0093-z.

Blum, K., Downs, B.W., Dushaj, K., Li, M., Braverman, E.R., Fried, L., Waite, R., Demetrovics, Z., \& Badgaiyan, R.D. (2016). The benefits of customized DNA directed nutrition to balance the rain reward circuitry and reduce addictive behaviors. Precision Medicine Journal, 1(1): 18-33.

Blum, K., Febo, M., McLaughlin, T., Cronjé, F.J., Han, D., \& Gold, S.M. (2014). Hatching the behavioral addiction egg: Reward Deficiency Solution System (RDSS) ${ }^{\mathrm{TM}}$ as a function of dopaminergic neurogenetics and brain functional connectivity linking all addictions under a common 
rubric. J Behav Addict., 3(3):149-56. doi: 10.1556/JBA.3.2014.019

Blum, K., Febo, M., \& Badgaiyan, R.D. (2016). Fifty Years in the development of a GlutaminergicDopaminergic Optimization Complex (KB220) to balance brain reward circuitry in Reward Deficiency Syndrome: A pictorial. Austin Addict Sci., 1(2). pii: 1006.

Blum, K., Febo, M., Badgaiyan, R.D., Demetrovics, Z., Simpatico, T., Fahlke, C., Oscar-Berman, M., Li, M., Dushaj, K., \& Gold, M.S. (2016). Common neurogenetic diagnosis and meso-limbic manipulation of hypodopaminergic function in Reward Deficiency Syndrome (RDS): Changing the recovery landscape. Curr Neuropharmacol., 15 (1): 184-194.

Blum, K., Gardner, E., Oscar-Berman, M., Gold, M. (2012). "Liking" and "wanting" linked to Reward Deficiency Syndrome (RDS): Hypothesizing differential responsivity in brain reward circuitry. Curr Pharm Des., 18(1):113-8.

Blum, K., \& Gold, M.S. (2011). Neurochemical activation of brain reward meso-limbic circuitry is associated with relapse prevention and drug hunger: A hypothesis. Med Hypotheses., 76(4):576-84. doi: 10.1016/j.mehy.2011.01.005.

Blum, K., Noble, E.P., Sheridan, P.J., Montgomery, A., Ritchie, T., Jagadeeswaran, P., Nogami H., Briggs, A.H., \& Cohn, J.B. (1990). Allelic association of human dopamine D2 receptor gene in alcoholism. JAMA, 263(15):2055-60.

Blum, K., Smolen, A., Haberstick, B.C., Smith, D.E., Febo, M., Simpatico, T., Han, D., Villapiano, A., Femino, J., \& Gold, M.S. (2014). Genetic Addiction Risk Score $\left(\mathbf{G A R S}_{\mathbf{R X}^{\mathrm{TM}}}\right.$, Comprehensive Analysis of Reported Drugs (CARD ${ }^{\text {TM}}$ ) dopamine agonist Therapy (KB220z ${ }^{\mathrm{TM}}$ ) embracing genetic prediction of addiction risk severity for pain treatment. ARSA Pain Meeting, San Francisco, California: American Society of Regional Anesthesia and Pain Medicine.

Blum, K., Thanos, P.K., Badgaiyan, R.D., Febo, M., Oscar-Berman, M., Fratantonio, J., Demotrovics, Z., \& Gold, M.S. (2015). Neurogenetics and gene therapy for reward deficiency syndrome: are we going to the Promised Land? Expert Opin Biol Ther., 15(7):973-85. doi: 10.1517/14712598.2015.1045871.

Blum, K., Wood, R.C., Braverman, E.R., Chen, T.J., \& Sheridan, P.J. (1995). The $\mathrm{D} 2$ dopamine receptor gene as a predictor of compulsive disease: Bayes' theorem. Funct Neurol., 10(1):37-44.

Borsook, D., Linnman, C., Faria, V., Strassman, A.M., Becerra, L., Elman, I. (2016). Reward deficiency and anti-reward in pain chronification. Neurosci Biobehav Rev., 68:282-97. doi:10.1016/j.neubiorev.2016.05.033.

Center for Behavioral Health Statistics and Quality (CBHSQ) (2013). 2012 national survey on drug use and health: Detailed tables. Substance 
Abuse and Mental Health Services Administration, Rockville, MD.

CDC. (2013). Vital signs. Prescription Painkiller Overdoses: A Growing Epidemic, Especially Among Women. Retrieved from URL: http://www.cdc.gov/vitalsigns/Prescri ptionPainkillerOverdoses/index.html

Damez-Werno, D.M., Sun, H., Scobie, K.N., Shao, N, Rabkin, J., Dias, C., Calipari, E.S., Maze I., Pena, C.J., Walker, D.M., Cahill, M.E., Chandra, R., Gancarz, A., Mouzon, E., Landry, J.A., Cates, H., Lobo, M.K., Dietz, D., Allis, C.D., Guccione, E., Turecki, G., Defilippi, P., Neve, R.L., Hurd, Y.L., Shen, L., \& Nestler, E.J. (2016). Histone arginine methylation in cocaine action in the nucleus accumbens. Proc Natl Acad Sci USA, 113(34):9623-8. doi: 10.1073/pnas.1605045113.

Eisenstein, S.A., Bogdan, R., Chen, L., Moerlein, S.M., Black, K.J., Perlmutter, J.S., Hershey T, Barch, D.M. (2016). Preliminary evidence that negative symptom severity relates to multilocus genetic profile for dopamine signaling capacity and D2 receptor binding in healthy controls and in schizophrenia. $J$ Psychiatr Res.; 86:917.doi:10.1016/j.jpsychires.2016.11. 007.

Galvan, L., André, V.M., Wang, E.A., Cepeda, C., \& Levine, M.S. (2012). Functional differences between direct and indirect striatal output pathways in Huntington's disease. $J$ Huntingtons Dis., 1(1):17-25. doi: 10.3233/JHD-2012-120009.
Gokcumen, O., Zhu, Q., Mulder, L.C., Iskow, R.C., Austermann, C., Scharer, C.D., Raj, T., Boss, J.M., Sunyaev, S., Price, A., Stranger, B., Simon, V., \& Lee, C. (2013). Balancing selection on a regulatory region exhibiting ancient variation that predates human- neandertal divergence. PLoS Genet., 9(4):e1003404. doi: 10.1371/journal. pgen. 1003404 .

Gold, M.S., Blum, K., Oscar-Berman, M., \& Braverman, E.R. (2014). Low dopamine function in attention deficit/hyperactivity disorde $r$ : should genotyping signify early diagnosis in children? Postgrad Med., 126(1):153-77. doi: 10.3810/pgm.2014.01.2735.

Mattoo, S. (2009). Prevalence and correlates of epileptic seizure in substanceabusing subjects. Psychiatry Clin Neurosci., 63(4):580-2.

Modestino, E.J., Blum, K., Oscar-Berman, M., Gold, M.S., Duane, D.D., Sultan, S.G.S., \& Auerbach, S. H. (2015). Reward Deficiency Syndrome: Attentional/arousal subtypes, limitations of current diagnostic nosology, and future research. $J$ Reward Defic Syndr. 1(1): 6-9.

Moore, A., Derry, S., Eccleston, C. \& Kalso, E. (2013). Expect analgesic failure; pursue analgesic success. BMJ, 346 (7911), f2690.

Noble, E.P. (2003). D2 dopamine receptor gene in psychiatric and neurologic disorders and its phenotypes. Am $J$ Med Genet B Neuropsychiatr Genet., 116B(1):103-25. 
Noble, E.P., Blum, K., Ritchie, T., Montgomery, A., \& Sheridan, P.J. (1991). Allelic association of the D2 dopamine receptor gene with receptor-binding characteristics in alcoholism. Arch Gen Psychiatry, 48(7):648-54.

Paulozzi L.J., Budnitz, D.S., \& Xi, Y. (2006). Increasing deaths from opioid analgesics in the United States. Pharmacoepidemiol Drug Saf., 15(9):618-27.

Plomin, R., Owen, M.J., \& McGuffin, P. (1994). The genetic basis of complex human behaviors. Science, 264(5166):1733-9.

Pohjalainen, T., Rinne, J.O., Någren, K., Lehikoinen, P., Anttila, K., Syvälahti, E.K., \& Hietala, J. (1998). The A1 allele of the human D2 dopamine receptor gene predicts low D2 receptor availability in healthy volunteers. Mol. Psychiatry, 3(3):256-60.

Muhuri, P.K. Gfroerer, J.C., \& Davies, M.C. (2013). Associations of nonmedical pain reliever use and initiation of heroin use in the United States. US. Center for behavioral Health Statistics and QualityData Review. SAMHSA. URL: http://www.samhsa. gov/data/2k13/DataReview/DR006/n onmedical-pain-reliever-use2013.htm

Skolnick, P., \& Volkow, N.D. (2016) Reenergizing the development of pain therapeutics in light of the Opioid
Epidemic. Neuron, 92(2):294-297. doi: 10.1016/j.neuron.2016.09.051.

Substance Abuse and Mental Health Services Administration (SAMHSA) (2013). Results from the 2012 national survey on drug use and health: Summary of national findings. NSDUH Series $\mathrm{H}-46$, $H H S$ Publication No. (SMA) 13-4795. Rockville, MD.

Thanos, P.K., Michaelides, M., Umegaki, H., \& Volkow, N.D. (2008). D2R DNA transfer into the nucleus accumbens attenuates cocaine selfadministration in rats. Synapse, 62(7):481-6. doi: 10.1002/syn.20523.

Thanos, P.K., Rivera, S.N., Weaver, K., Grandy, D.K., Rubinstein, M., Umegaki, H., Wang, G.J., Hitzemann, R., \& Volkow, N.D. (2005). Dopamine D2R DNA transfer in dopamine D2 receptordeficient mice: effects on ethanol drinking. Life Sci., 77(2):130-9.

UNODC (2012). World Drug Report 2012.United Nations publication, Sales No. E.12.XI.1. Yamaguchi, N., Shibazaki, M., Yamada, C., Anzai, E., Morii, M., Nakayama, Y., Kuga, T.,Hashimoto, Y., Tomonaga, T., \& Yamaguchi, N. (2016). Tyrosine phosphorylation of The Pioneer Transcription Factor FoxA1 promotes activation of estrogen signaling. J Cell Biochem., [Epub ahead of print] doi: $10.1002 /$ jcb. 25804 . 